Comment. Math. Helv. 78 (2003) 865-883 0010-2571/03/040865-19

DOI $10.1007 / \mathrm{s} 00014-003-0775-8$
(C) 2003 Birkhäuser Verlag, Basel

Commentarii Mathematici Helvetici

\title{
Some geometric properties of the Bakry-Émery-Ricci tensor
}

John Lott

\begin{abstract}
The Bakry-Émery tensor gives an analog of the Ricci tensor for a Riemannian manifold with a smooth measure. We show that some of the topological consequences of having a positive or nonnegative Ricci tensor are also valid for the Bakry-Émery tensor. We show that the Bakry-Émery tensor is nondecreasing under a Riemannian submersion whose fiber transport preserves measures up to constants. We give some relations between the Bakry-Émery tensor and measured Gromov-Hausdorff limits.
\end{abstract}

Mathematics Subject Classification (2000). Primary 53C21; Secondary 58G25.

Keywords. Ricci tensor, metric-measure space, Riemannian submersion.

\section{Introduction}

When considering the metric structure of manifolds with lower Ricci curvature bounds, it is natural to carry along the extra structure of a measure and consider metric-measure spaces. This is especially relevant for collapsing, and has been discussed by Cheeger-Colding [8, 9, 10], Fukaya [15] and Gromov [17, Chapter $\left.3 \frac{1}{2}\right]$.

In this paper we consider smooth metric-measure spaces. Let $M$ be an $n$ dimensional Riemannian manifold, with metric $g$. Let $d \mathrm{vol}_{M}$ denote the Riemannian density on $M$. Let $\phi$ be a smooth positive function on $M$. Then $\left(M, \phi d \mathrm{vol}_{M}\right)$ is a smooth metric-measure space. For reasons coming from the study of diffusion processes, Bakry and Émery [4] defined a generalization of the Ricci tensor of $M$ by

$$
\widetilde{\operatorname{Ric}}_{\infty}=\operatorname{Ric}-\operatorname{Hess}(\ln \phi) .
$$

In terms of indices, $\left(\widetilde{\operatorname{Ric}}_{\infty}\right)_{\alpha \beta}=\operatorname{Ric}_{\alpha \beta}-(\ln \phi)_{; \alpha \beta}$.

It turns out that the Bakry-Émery tensor (1.1) has interesting connections to logarithmic Sobolev inequalities, isoperimetric inequalities and heat semigroups. We refer to [2] and [19] for information on these connections. (In fact, Bakry and Émery defined their tensor in a more abstract setting than what we consider.)

Research supported by NSF grant DMS-0072154. 
We are interested in the geometric implications of bounds on the Bakry-Émery tensor. As in [20], let us define a related tensor $\widetilde{\operatorname{Ric}}_{q}$. Given $q \in(0, \infty)$, put

$$
\begin{aligned}
\widetilde{\operatorname{Ric}}_{q} & =\operatorname{Ric}-\operatorname{Hess}(\ln \phi)-\frac{1}{q} d \ln \phi \otimes d \ln \phi \\
& =\operatorname{Ric}-\frac{\operatorname{Hess}(\phi)}{\phi}+\left(1-\frac{1}{q}\right) \frac{d \phi}{\phi} \otimes \frac{d \phi}{\phi} \\
& =\operatorname{Ric}-q \frac{\operatorname{Hess}\left(\phi^{\frac{1}{q}}\right)}{\phi^{\frac{1}{q}}} .
\end{aligned}
$$

Clearly, if $\widetilde{\operatorname{Ric}}_{q} \geq r g$ then $\widetilde{\operatorname{Ric}}_{\infty} \geq r g$. In the terminology of [3], a condition of the form $\widetilde{\operatorname{Ric}}_{q} \geq r g$ implies a curvature-dimension inequality $\operatorname{CD}(r, n+q)$.

Our first result extends some classical topological results about the Ricci tensor (i.e. when $\phi$ is constant) to the setting of the Bakry-Émery tensor.

Theorem 1. Suppose that $M$ is connected and closed.

1. If $\widetilde{\mathrm{Ric}}_{\infty}>0$ then $\pi_{1}(M)$ is finite.

2. If $\widehat{\operatorname{Ric}}_{q} \geq 0$ and $q \in(0, \infty)$ then $\pi_{1}(M)$ has a finite-index free abelian subgroup of rank at most $n$.

3. If $\widehat{\operatorname{Ric}}_{\infty} \geq 0$ then $\mathrm{H}^{1}(M ; \mathbb{R})$ is isomorphic to the linear space of parallel 1 -forms on $M$ whose pairing with $\operatorname{grad}(\phi)$ vanishes identically. In particular, if $\widetilde{\operatorname{Ric}}_{\infty} \geq 0$ then $\mathrm{b}_{1}(M) \leq n$. If $\widetilde{\operatorname{Ric}}_{\infty} \geq 0$ and $\mathrm{b}_{1}(M)=n$ then $M$ is a flat torus and $\phi$ is constant.

4. If $\widehat{\mathrm{Ric}}_{\infty}<0$ then the isometry group of $(M, g)$ is finite.

5. If $\widetilde{\mathrm{Ric}}_{\infty} \leq 0$ then any Killing vector field on $(M, g)$ is parallel and annihilates $\phi$.

Remark. Theorem 1.2 is a strengthening of [20, Theorem 6], which says that if $\widetilde{\operatorname{Ric}}_{q} \geq 0$ and $q \in(0, \infty)$ then $\pi_{1}(M)$ has polynomial growth of order at most $n+q$.

The proofs of parts $3-5$ of Theorem 1 use a Bochner-type identity. If the pair $(g, \phi)$ is only $C^{0} \cap H^{1}$-regular then one can use this identity to still make sense of the notion $\widetilde{\operatorname{Ric}}_{\infty} \geq r g$ or $\widetilde{\operatorname{Ric}}_{q} \geq r g$ (see Definition 1 of Section 2).

An important result in the study of manifolds of nonnegative sectional curvature is O'Neill's theorem, which says that sectional curvature is nondecreasing under a Riemannian submersion [7, Chapter 9]. We show that there is a Ricci analog of O'Neill's theorem, provided that one uses the Bakry-Émery tensor and assumes that the fiber transport of the Riemannian submersion preserves measures up to multiplicative constants.

Suppose that a Riemannian submersion $p: M \rightarrow B$ has compact fiber $F$. Put $F_{b}=p^{-1}(b)$. Given a smooth curve $\gamma:[0,1] \rightarrow B$ and a point $m \in F_{\gamma(0)}$, let $\rho(m)$ be the endpoint $\bar{\gamma}(1)$ of the horizontal lift $\bar{\gamma}$ of $\gamma$ that starts at $\bar{\gamma}(0)$. Then $\rho$ is the fiber transport diffeomorphism from $F_{\gamma(0)}$ to $F_{\gamma(1)}$. 
Given the positive function $\phi^{M}$ on $M$, define $\phi^{B}$, a smooth positive function on $B$, by

$$
p_{*}\left(\phi^{M} d \operatorname{vol}_{M}\right)=\phi^{B} d \operatorname{vol}_{B} .
$$

Let $\widetilde{\operatorname{Ric}}_{\infty}^{M}$ and $\widetilde{\operatorname{Ric}}_{\infty}^{B}$ denote the corresponding Bakry-Émery tensors. Let $d \mathrm{vol}_{F}$ denote the fiberwise Riemannian density.

Theorem 2. Suppose that fiber transport preserves the fiberwise measure $\phi_{M} d \mathrm{vol}_{F}$ up to a multiplicative constant, i.e. for any smooth curve $\gamma:[0,1] \rightarrow B$, there is a constant $c_{\gamma}>0$ such that $\rho^{*}\left(\left.\phi^{M}\right|_{F_{\gamma(1)}} d \operatorname{vol}_{F_{\gamma(1)}}\right)=\left.c_{\gamma} \phi^{M}\right|_{F_{\gamma(0)}} d \operatorname{vol}_{F_{\gamma(0)}}$.

1. For any $r \in \mathbb{R}$, if $\widetilde{\operatorname{Ric}}_{\infty}^{M} \geq r g^{M}$ then $\widetilde{\operatorname{Ric}}_{\infty}^{B} \geq r g^{B}$.

2. Suppose in addition that $\phi^{M}=1$. Put $q=\operatorname{dim}(F)$. For any $r \in \mathbb{R}$, if $\operatorname{Ric}^{M} \geq r g^{M}$ then $\widetilde{\operatorname{Ric}}_{q}^{B} \geq r g^{B}$.

Using Theorem 2, we show a relationship between $\widetilde{\operatorname{Ric}_{q}}$ and collapsing.

Theorem 3. 1. Given $r \in \mathbb{R}$ and an integer $q \geq 2$, let $(B, \phi)$ be a smooth closed measured Riemannian manifold with $\widetilde{\operatorname{Ric}}_{q}^{B} \geq r g^{B}$. Then $(B, \phi)$ is the measured Gromov-Hausdorff limit of a sequence of $(n+q)$-dimensional closed Riemannian manifolds $\left(M_{i}, g_{i}\right)$ with $\operatorname{Ric}\left(M_{i}, g_{i}\right) \geq r g_{i}$.

2. Let $\left\{\left(M_{i}, g_{i}\right)\right\}_{i=1}^{\infty}$ be a sequence of $N$-dimensional connected closed Riemannian manifolds with sectional curvatures bounded above in absolute value by $\Lambda$ and diameters bounded above by $D$, for some $D, \Lambda \in \mathbb{R}^{+}$. Let $(X, \mu)$ be a limit point for $\left\{\left(M_{i}, g_{i}\right)\right\}_{i=1}^{\infty}$ in the measured Gromov-Hausdorff topology. Suppose that for some $r \in \mathbb{R}$ and all $i \in \mathbb{Z}^{+}, \operatorname{Ric}\left(M_{i}, g_{i}\right) \geq r g_{i}$. Suppose that $X$ is an $n$-dimensional closed manifold. Put $q=N-n$.

a. If $q=0$ then $X$ has Ric $\geq r g$ in the generalized sense of Definition 1 below.

b. If $q>0$ then $X$ has $\widetilde{\operatorname{Ric}}_{q} \geq \operatorname{rg}$ in the generalized sense of Definition 1 below.

Finally, we give a condition in terms of distances and masses that is equivalent to having Bakry-Émery tensor bounded below by $r$. If $\mathcal{O}$ is a measurable subset of $M$, put

$$
\operatorname{vol}_{\phi}(\mathcal{O})=\int_{\mathcal{O}} \phi d \operatorname{vol}_{M}
$$

Following [17, Section 5.45], we define the notion of a distance tube in $M$. Let $T_{0}$ be a closed subset of $M$. A subset $T \subset M$ containing $T_{0}$ is a distance tube with base $T_{0}$ if for all $t \in T$, there is a segment $s \subset T$ from some $t_{0} \in T_{0}$ to $t$ with length $l(s)=d\left(t, T_{0}\right)$. For $0<u_{1}<u_{2}$, define the distance annulus

$$
A\left(u_{1}, u_{2}\right)=\left\{t \in T: u_{1} \leq d\left(t, T_{0}\right) \leq u_{2}\right\} .
$$


Given $c \in \mathbb{R}$, put

$$
\widehat{v}\left(u_{1}, u_{2}, c\right)=\int_{u_{1}}^{u_{2}} e^{-\frac{r}{2} x^{2}+c x} d x
$$

Theorem 4. Suppose that $\widetilde{\operatorname{Ric}}_{\infty}(M, g, \phi) \geq r g$ for some $r \in \mathbb{R}$. Given numbers $0<u_{1}<u_{2}<u_{3}$, we assume that the tube $T$ is a disjoint union of segments $s$, starting at $T_{0}$, of length at least $u_{3}$. We also assume that $\operatorname{vol}_{\phi}\left(A\left(u_{2}, u_{3}\right)\right)>0$. Suppose that for some $c \in \mathbb{R}$,

$$
\frac{\operatorname{vol}_{\phi}\left(A\left(u_{2}, u_{3}\right)\right)}{\operatorname{vol}_{\phi}\left(A\left(u_{1}, u_{2}\right)\right)} \leq \frac{\widehat{v}\left(u_{2}, u_{3}, c\right)}{\widehat{v}\left(u_{1}, u_{2}, c\right)} .
$$

Then there is a subtube $T^{\prime} \subset T$ consisting of a union of segments $s$ from $T_{0}$, such that

1.

$$
\frac{\operatorname{vol}_{\phi}\left(T^{\prime} \cap A\left(u_{1}, u_{2}\right)\right)}{\operatorname{vol}_{\phi}\left(A\left(u_{1}, u_{2}\right)\right)} \geq 1-\frac{\operatorname{vol}_{\phi}\left(A\left(u_{2}, u_{3}\right)\right)}{\operatorname{vol}_{\phi}\left(A\left(u_{1}, u_{2}\right)\right)}\left(\frac{\widehat{v}\left(u_{2}, u_{3}, c\right)}{\widehat{v}\left(u_{1}, u_{2}, c\right)}\right)^{-1},
$$

2. If a segment $s \subset T$, starting from $T_{0}$, intersects $T^{\prime} \cap A\left(u_{2}, u_{3}\right)$ then $s \subset T^{\prime}$, and

3. For all $u_{4}>u_{3}$

$$
\frac{\operatorname{vol}_{\phi}\left(T^{\prime} \cap A\left(u_{3}, u_{4}\right)\right)}{\operatorname{vol}_{\phi}\left(T^{\prime} \cap A\left(u_{2}, u_{3}\right)\right)} \leq \frac{\widehat{v}\left(u_{3}, u_{4}, c\right)}{\widehat{v}\left(u_{2}, u_{3}, c\right)} .
$$

Conversely, suppose that there is a number $r \in \mathbb{R}$ so that for each tube $T$ and $c \in \mathbb{R}$ satisfying (1.7), there is a subtube $T^{\prime}$ with the above properties. Then $\widetilde{\operatorname{Ric}}_{\infty}(M, g, \phi) \geq r g$.

In Sections 2-5 we prove Theorems 1-4, respectively. In Section 6 we make some remarks.

I thank Max Karoubi for his hospitality at the Université de Paris VII, and Thierry Coulhon and Sasha Grigor'yan for their hospitality at the Institut Henri Poincaré, while part of this research was performed.

\section{Proof of Theorem 1}

We first prove parts 1 and 2 of the theorem. If $\widetilde{\operatorname{Ric}}_{\infty}>0$ then $\widetilde{\operatorname{Ric}}_{q}>0$ for some $q \in(0, \infty)$. Increasing $q$ if necessary, we may assume without loss of generality that $q$ is an integer greater than one. Thus for parts 1 and 2 , it is enough to consider the case when $\widetilde{\operatorname{Ric}}_{q}>0$ or $\widetilde{\operatorname{Ric}}_{q} \geq 0$, for some integer $q$ greater than one.

Given $i \in \mathbb{Z}^{+}$, consider $S^{q} \times M$ with the warped product metric $g^{S^{q} \times M}=$ $g^{M}+i^{-2} \phi^{\frac{2}{q}} g^{S^{q}}$. Let $p: S^{q} \times M \rightarrow M$ be the projection. Let $\bar{X}$ be the horizontal lift to $S^{q} \times M$ of a vector field $X$ on $M$ and let $\bar{U}$ be a vertical vector field on 
$S^{q} \times M$. From [7, Proposition 9.106],

$$
\begin{aligned}
& \operatorname{Ric}^{S^{q} \times M}(\bar{X}, \bar{X})=p^{*}\left(\operatorname{Ric}^{M}(X, X)-q \frac{\operatorname{Hess}\left(\phi^{\frac{1}{q}}\right)(X, X)}{\phi^{\frac{1}{q}}}\right) \\
& \operatorname{Ric}^{S^{q} \times M}(\bar{X}, \bar{U})=0 \\
& \operatorname{Ric}^{S^{q} \times M}(\bar{U}, \bar{U})=\operatorname{Ric}^{S^{q}}(\bar{U}, \bar{U})+(\bar{U}, \bar{U}) p^{*}\left(-\frac{\nabla^{2} \phi^{\frac{1}{q}}}{\phi^{\frac{1}{q}}}-(q-1) \frac{\left|\nabla \phi^{\frac{1}{q}}\right|^{2}}{\phi^{\frac{2}{q}}}\right) .
\end{aligned}
$$

Taking $i \rightarrow \infty$, we see that if $\widetilde{\operatorname{Ric}_{q}}(M, g, \phi) \geq r g$ then $\left(M, g^{M}, \phi\right)$ is the limit of a sequence of $(n+q)$-dimensional manifolds with Ricci curvature bounded below by $r$. If $r$ is positive then from Myers' theorem, $\pi_{1}\left(S^{q} \times M\right) \cong \pi_{1}(M)$ is finite. This proves part 1 of the theorem.

Now suppose that $r \geq 0$. For $i$ large, the warped product metric on $S^{q} \times M$ has nonnegative Ricci curvature. There is a $k \geq 0$ so that $\pi_{1}\left(S^{q} \times M\right) \cong \pi_{1}(M)$ has a finite-index free abelian subgroup of rank $k$ and the universal cover $S^{q} \times \widetilde{M}$ has an isometric splitting as $\mathbb{R}^{k} \times Y^{n+q-k}$, where $Y$ is closed and simply-connected [12]. Considering the cohomology groups of $S^{q} \times \widetilde{M} \cong \mathbb{R}^{k} \times Y^{n+q-k}$, it follows that

$$
q+\max \left\{j: \mathrm{H}^{j}(\widetilde{M} ; \mathbb{Z}) \neq 0\right\}=n+q-k .
$$

Then $k=n-\max \left\{j: \mathrm{H}^{j}(\widetilde{M} ; \mathbb{Z}) \neq 0\right\} \leq n$, which proves part 2 of the theorem.

To prove the rest of the theorem, if $V$ is a vector field on $M$, let $V^{\sharp}$ denote the dual 1-form. If $\omega$ is a 1 -form on $M$, let $\omega_{\sharp}$ denote the dual vector field. Let $i_{V}$ denote interior multiplication with respect to $V$ and let $\mathcal{L}_{V}$ denote Lie differentiation with respect to $V$.

If $T$ is a tensor field on $M$, let $(T, T) \in C^{\infty}(M)$ be the inner product coming from the Riemannian metric $g$. Put

$$
\langle T, T\rangle=\int_{M}(T, T)(m) \phi(m) d \operatorname{vol}_{M}(m) .
$$

Let $\left(\Omega^{*}(M), d\right)$ denote the de Rham complex of $M$. Let $\delta$ be the formal adjoint of $d$ with respect to the Riemannian metric $g$, i.e. in the case $\phi=1$, and let $\widetilde{\delta}$ be the formal adjoint of $d$ with respect to $\langle\cdot, \cdot\rangle$. Then

$$
\widetilde{\delta}=\delta-i_{(d \ln \phi)_{\sharp}} .
$$

Put $\triangle=d \delta+\delta d$ and $\widetilde{\triangle}=d \widetilde{\delta}+\widetilde{\delta} d$. Then

$$
\widetilde{\triangle}=\triangle-d i_{(d \ln \phi)_{\sharp}}-i_{(d \ln \phi)_{\sharp}} d=\triangle-\mathcal{L}_{(d \ln \phi)_{\sharp}} .
$$

The Bochner identity says that if $\omega$ is a 1-form then there is an equality of functions on $M$ :

$$
\frac{1}{2} \delta d(\omega, \omega)=(\omega, \triangle \omega)-(\nabla \omega, \nabla \omega)-(\omega, \operatorname{Ric} \omega) .
$$


On the other hand,

$$
\frac{1}{2} i_{(d \ln \phi)_{\sharp}} d(\omega, \omega)=\frac{1}{2} \mathcal{L}_{(d \ln \phi)_{\sharp}}(\omega, \omega) .
$$

We have

$$
\mathcal{L}_{(d \ln \phi)_{\sharp}} g=2 \operatorname{Hess}(\ln \phi) .
$$

Then

$$
\frac{1}{2} i_{(d \ln \phi)_{\sharp}} d(\omega, \omega)=\left(\omega, \mathcal{L}_{(d \ln \phi)_{\sharp}} \omega\right)-(\omega, \operatorname{Hess}(\ln \phi) \omega) .
$$

(The minus sign in (2.9) comes from the fact that the pairing is on 1-forms instead of vector fields.) Equations (2.4), (2.5), (2.6) and (2.9) give

$$
\frac{1}{2} \widetilde{\delta} d(\omega, \omega)=(\omega, \widetilde{\triangle} \omega)-(\nabla \omega, \nabla \omega)-\left(\omega, \widetilde{\operatorname{Ric}}_{\infty} \omega\right)
$$

Multiplying (2.10) by $\phi$ and integrating over $M$, we obtain

$$
0=\langle\omega, \widetilde{\triangle} \omega\rangle-\langle\nabla \omega, \nabla \omega\rangle-\left\langle\omega, \widetilde{\operatorname{Ric}}_{\infty} \omega\right\rangle
$$

or

$$
\langle d \omega, d \omega\rangle+\langle\widetilde{\delta} \omega, \widetilde{\delta} \omega\rangle-\langle\nabla \omega, \nabla \omega\rangle=\left\langle\omega, \widetilde{\operatorname{Ric}}_{\infty} \omega\right\rangle
$$

We can apply usual elliptic theory to the de Rham complex, with the inner product $\langle\cdot, \cdot\rangle$, to obtain an isomorphism

$$
\mathrm{H}^{*}(M ; \mathbb{R}) \cong\left\{\omega \in \Omega^{*}(M): d \omega=\widetilde{\delta} \omega=0\right\} .
$$

If $\widetilde{\operatorname{Ric}}_{\infty} \geq 0$ and a 1 -form $\omega$ satisfies $d \omega=\widetilde{\delta} \omega=0$ then (2.12) implies that $\nabla \omega=0$. Hence $\delta \omega=0$. Along with $\widetilde{\delta} \omega=0,(2.4)$ now implies that $\omega(\operatorname{grad}(\phi))=$ 0 . Conversely, if $\nabla \omega=\omega(\operatorname{grad}(\phi))=0$ then $d \omega=\widetilde{\delta} \omega=0$. This proves the isomorphism in part 3 of the theorem.

If $\mathrm{b}_{1}(M)=n$ then there are $n$ linearly-independent parallel 1-forms on $M$ that annihilate $\operatorname{grad}(\phi)$. The usual argument shows that $M$ is a flat torus. As the parallel 1-forms on $M$ annihilate $\operatorname{grad}(\phi), \phi$ must be constant. This proves part 3 of the theorem.

A pointwise algebraic computation shows that

$$
(d \omega, d \omega)+\left(\mathcal{L}_{\omega_{\sharp}} g, \mathcal{L}_{\omega_{\sharp}} g\right)=2(\nabla \omega, \nabla \omega) .
$$

Then (2.12) becomes

$$
\langle\nabla \omega, \nabla \omega\rangle+\langle\widetilde{\delta} \omega, \widetilde{\delta} \omega\rangle-\left\langle\omega, \widetilde{\operatorname{Ric}}_{\infty} \omega\right\rangle=\left\langle\mathcal{L}_{\omega_{\sharp}} g, \mathcal{L}_{\omega_{\sharp}} g\right\rangle .
$$

If $\widetilde{\operatorname{Ric}}_{\infty}<0$ and $\mathcal{L}_{V} g=0$ then taking $\omega=V^{\sharp},(2.15)$ implies that $V=0$. Hence the isometry group of $(M, g)$ is discrete and, being compact, must be finite. This proves part 4 of the theorem. 
If $\widetilde{\operatorname{Ric}}_{\infty} \leq 0$ and $\mathcal{L}_{V} g=0$ then (2.15) implies that $\nabla V^{\sharp}=\widetilde{\delta} V^{\sharp}=0$. As before, we obtain that $V \phi=0$. This proves part 5 of the theorem.

Remarks. 1. If we put $\omega=d f$ in $(2.10)$ then we recover the definition of $\widetilde{\operatorname{Ric}}_{\infty}$ from [4].

2. Jianguo Cao pointed out to me that a formula related to (2.12) has been used to study the $\bar{\partial}$-operator on complete Kähler manifolds [14, Théorème 5.1].

3. The operator $\widetilde{\Delta}$ is related to the Witten Laplacian of [22], but the two operators are distinct. To see the relation, note that $\widetilde{\delta}=\phi^{-1} \delta \phi$. Put $D=\phi^{\frac{1}{2}} d \phi^{-\frac{1}{2}}$ and $D^{*}=\phi^{-\frac{1}{2}} \delta \phi^{\frac{1}{2}}$. Then the Witten Laplacian $D D^{*}+D^{*} D$ is related to $\widetilde{\Delta}$ by

$$
D D^{*}+D^{*} D=\phi^{\frac{1}{2}} \widetilde{\Delta} \phi^{-\frac{1}{2}} \text {. }
$$

The Bochner-type identity $(2.12)$, when translated to a statement about $D D^{*}+$ $D^{*} D$, becomes

$$
D D^{*}+D^{*} D=\left(\phi^{\frac{1}{2}} \nabla \phi^{-\frac{1}{2}}\right)^{*}\left(\phi^{\frac{1}{2}} \nabla \phi^{-\frac{1}{2}}\right)+\widetilde{\operatorname{Ric}}_{\infty},
$$

where the adjoints are with respect to the unweighted $L^{2}$-inner product. In contrast, in Morse-Witten theory one collects the terms differently, by writing $D D^{*}+D^{*} D=\nabla^{*} \nabla+\ldots$

4. The equality $(2.12)$ gives a way of defining the notion of $\widetilde{\operatorname{Ric}}_{\infty} \geq r g$ for a class of nonsmooth measured manifolds $(M, g, \phi)$. Namely, suppose that $M$ is a manifold whose transition maps are $C^{1,1}$-regular. Let $g$ be a Riemannian metric on $M$ whose components, in local charts, are in $C^{0} \cap H^{1}$, where $H^{1}$ denotes the Sobolev space. Let $\phi \in C^{0}(M) \cap H_{\text {loc }}^{1}(M)$ be a positive function. (There are a smooth manifold $M^{\prime}$ and a $C^{1,1}$-diffeomorphism $M^{\prime} \rightarrow M$. Hence after pulling back, if one wants then one can assume that $g$ and $\phi$ are defined on a smooth manifold.)

Definition 1. We say that $\operatorname{Ric}(M, g) \geq r g$ if for all compactly-supported Lipschitzregular 1-forms $\omega$ on $M$,

$\int_{M}(d \omega, d \omega) d \operatorname{vol}_{M}+\int_{M}(\delta \omega, \delta \omega) d \operatorname{vol}_{M}-\int_{M}(\nabla \omega, \nabla \omega) d \operatorname{vol}_{M} \geq r \int_{M}(\omega, \omega) d \operatorname{vol}_{M}$.

We say that $\widetilde{\operatorname{Ric}}_{\infty}(M, g, \phi) \geq r g$ if for all compactly-supported Lipschitz-regular 1 -forms $\omega$ on $M$,

$$
\langle d \omega, d \omega\rangle+\langle\widetilde{\delta} \omega, \widetilde{\delta} \omega\rangle-\langle\nabla \omega, \nabla \omega\rangle \geq r\langle\omega, \omega\rangle .
$$

We say that $\widetilde{\operatorname{Ric}_{q}}(M, g, \phi) \geq r g$ if for all compactly-supported Lipschitz-regular 1 -forms $\omega$ on $M$,

$$
\langle d \omega, d \omega\rangle+\langle\widetilde{\delta} \omega, \widetilde{\delta} \omega\rangle-\langle\nabla \omega, \nabla \omega\rangle-\frac{1}{q} \int_{M}(\omega(\nabla \ln \phi))^{2} \phi d \operatorname{vol}_{M} \geq r\langle\omega, \omega\rangle .
$$


An immediate consequence of the definition is the following lemma.

Lemma 1. Let $M$ be a smooth closed manifold.

1. If $\left\{g_{i}\right\}_{i=1}^{\infty}$ is a sequence of smooth Riemannian metrics on $M$ with $\operatorname{Ric}\left(M, g_{i}\right)$ $\geq r g_{i}$, and $g_{i} \stackrel{C^{0} \cap H^{1}}{\longrightarrow} g$ for some $C^{0} \cap H^{1}$-regular metric $g$, then $\operatorname{Ric}(M, g) \geq r g$.

2. If $\left\{\left(g_{i}, \phi_{i}\right)\right\}_{i=1}^{\infty}$ is a sequence of smooth Riemannian metrics and smooth positive functions on $M$ with $\widetilde{\operatorname{Ric}}_{\infty}\left(M, g_{i}, \phi_{i}\right) \geq r g_{i}$, and $\left(g_{i}, \phi_{i}\right) \stackrel{C^{0} \cap H^{1}}{\longrightarrow}(g, \phi)$ for some $C^{0} \cap H^{1}$-regular pair $(g, \phi)$, then $\widetilde{\operatorname{Ric}}_{\infty}(M, g, \phi) \geq r g$.

3. If $\left\{\left(g_{i}, \phi_{i}\right)\right\}_{i=1}^{\infty}$ is a sequence of smooth Riemannian metrics and smooth positive functions on $M$ with $\widetilde{\operatorname{Ric}}_{q}\left(M, g_{i}, \phi_{i}\right) \geq r g_{i}$, and $\left(g_{i}, \phi_{i}\right) \stackrel{C^{0} \cap H^{1}}{\longrightarrow}(g, \phi)$ for some $C^{0} \cap H^{1}$-regular pair $(g, \phi)$, then $\widetilde{\operatorname{Ric}}_{q}(M, g, \phi) \geq r g$.

For example, let $\left\{\left(M_{i}, g_{i}\right)\right\}_{i=1}^{\infty}$ be a sequence of $n$-dimensional closed Riemannian manifolds with Ricci curvatures bounded below by $r \in \mathbb{R}$, injectivity radii bounded below by $i_{0} \in \mathbb{R}^{+}$and diameters bounded above by $D \in \mathbb{R}^{+}$. Then $\left\{\left(M_{i}, g_{i}\right)\right\}_{i=1}^{\infty}$ has a limit point $X$ in the Gromov-Hausdorff topology. From [1], $X$ is an $n$-dimensional closed manifold with a Riemannian metric $g$ that is $W^{1, p_{-}}$ regular for all $p \in[1, \infty)$. From the Sobolev embedding theorem, $g$ is also $C^{0, \alpha}$-regular for all $\alpha \in(0,1)$. After applying diffeomorphisms one has $W^{1, p_{-}}$ convergence of a subsequence of $\left\{\left(M_{i}, g_{i}\right)\right\}_{i=1}^{\infty}$ to $(X, g)$, and so $\operatorname{Ric}(X, g) \geq r g$ in the sense of Definition 1.

For another example, suppose that $M$ is a compact Kähler manifold with local complex coordinates $\left\{z^{\alpha}\right\}$ and metric $g_{\alpha \bar{\beta}}$. Its Ricci form, in local coordinates, is the $(1,1)$-form $-\frac{1}{2} \partial \bar{\partial} \ln \operatorname{det}(g)$. Now suppose that the $g_{\alpha \bar{\beta}}$ are only $C^{0} \cap H^{1}$-regular. The Kähler condition still makes sense distributionally, and the Ricci form makes sense as a closed $(1,1)$-current. Then $\operatorname{Ric}(M, g) \geq 0$ in the sense of Definition 1 if and only if $-\frac{1}{2} \partial \bar{\partial} \ln \operatorname{det}(g)$ is a positive current. (This last condition makes sense for a much larger class of $g$.)

\section{Proof of Theorem 2}

We (mostly) use the notation of [7, Chapter 9]. If $X$ is a vector field on $B$, let $\bar{X}$ be its horizontal lift to $M$. Let $N$ be the mean curvature vector field to the fibers $F$. Let $A$ be the curvature of the horizontal distribution. Let $T$ be the second fundamental form tensor of the fibers $F$. Let $\nabla^{M}$ be the covariant derivative operator on $M$ and let $\nabla^{B}$ be the covariant derivative operator on $B$. From $[7$, (9.36c)], there is an identity of functions on $M$ :

$$
\operatorname{Ric}^{M}(\bar{X}, \bar{X})=\operatorname{Ric}^{B}(X, X)-2\left(A_{\bar{X}}, A_{\bar{X}}\right)-(T \bar{X}, T \bar{X})+\left(\bar{X}, \nabla \frac{M}{X} N\right) .
$$

Given $b \in B$, let $\left\{\theta_{t}\right\}_{t \in(-\epsilon, \epsilon)}$ be the flow of $X$ as defined in a neighborhood of $b$ and for $t$ in some interval $(-\epsilon, \epsilon)$. Let $\left\{\bar{\theta}_{t}\right\}_{t \in(-\epsilon, \epsilon)}$ be the flow of $\bar{X}$ that covers $\theta_{t}$. 
It sends fibers to fibers diffeomorphically. Hence it makes sense to define $\mathcal{L}_{\bar{X}} d$ vol $_{F}$ by

$$
\left.\left(\mathcal{L}_{\bar{X}} d \operatorname{vol}_{F}\right)\right|_{F_{b}}=\left.\left.\frac{d}{d t}\right|_{t=0}\left(\bar{\theta}_{t}^{*} d \operatorname{vol}_{F}\right)\right|_{F_{b}} .
$$

With our conventions,

$$
\mathcal{L}_{\bar{X}} d \operatorname{vol}_{F}=-(\bar{X}, N) d \operatorname{vol}_{F}
$$

We have

$$
\phi^{B}=\int_{F} \phi^{M} d \operatorname{vol}_{F}
$$

Then

$$
\begin{aligned}
X \phi^{B} & =\mathcal{L}_{X} \phi^{B}=\mathcal{L}_{X} \int_{F} \phi^{M} d \operatorname{vol}_{F}=\int_{F} \mathcal{L}_{\bar{X}}\left(\phi^{M} d \operatorname{vol}_{F}\right) \\
& =\int_{F}\left(\bar{X} \phi^{M}-(\bar{X}, N) \phi^{M}\right) d \operatorname{vol}_{F}
\end{aligned}
$$

and

$$
\begin{aligned}
X X \phi^{B}= & \int_{F}\left[\bar{X}\left(\bar{X} \phi^{M}-(\bar{X}, N) \phi^{M}\right)-(\bar{X}, N)\left(\bar{X} \phi^{M}-(\bar{X}, N) \phi^{M}\right)\right] d \operatorname{vol}_{F} \\
= & \int_{F}\left[\overline{X X} \phi^{M}-\bar{X}(\bar{X}, N) \phi^{M}-2(\bar{X}, N) \bar{X} \phi^{M}+(\bar{X}, N)^{2} \phi^{M}\right] d \operatorname{vol}_{F} \\
= & \int_{F}\left[\frac{\overline{X X} \phi^{M}}{\phi^{M}}-\left(\nabla \frac{M}{X} \bar{X}, N\right)-\left(\bar{X}, \nabla \frac{M}{X} N\right)-\left(\frac{\bar{X} \phi^{M}}{\phi^{M}}\right)^{2}\right. \\
& \left.+\left(\frac{\bar{X} \phi^{M}}{\phi^{M}}-(\bar{X}, N)\right)^{2}\right] \phi^{M} d \operatorname{vol}_{F} .
\end{aligned}
$$

Using the fact that $\nabla_{\bar{X}}^{M} \bar{X}=\overline{\nabla_{X}^{B} X}[7,(9.25 \mathrm{~d})]$, it follows that

$$
\begin{aligned}
\operatorname{Hess}\left(\phi_{B}\right)(X, X)= & X X \phi^{B}-\left(\nabla_{X}^{B} X\right) \phi^{B} \\
= & \int_{F}\left[\frac{\operatorname{Hess}\left(\phi^{M}\right)(\bar{X}, \bar{X})}{\phi^{M}}-\left(\bar{X}, \nabla \frac{M}{X} N\right)-\left(\frac{\bar{X} \phi^{M}}{\phi^{M}}\right)^{2}\right. \\
& \left.+\left(\frac{\bar{X} \phi^{M}}{\phi^{M}}-(\bar{X}, N)\right)^{2}\right] \phi^{M} d \operatorname{vol}_{F} \\
= & \int_{F}\left[\operatorname{Hess}\left(\ln \phi^{M}\right)(\bar{X}, \bar{X})-\left(\bar{X}, \nabla \frac{M}{X} N\right)\right. \\
& \left.+\left(\frac{\bar{X} \phi^{M}}{\phi^{M}}-(\bar{X}, N)\right)^{2}\right] \phi^{M} d \operatorname{vol}_{F} .
\end{aligned}
$$


Substituting $\left(\bar{X}, \nabla \frac{M}{X} N\right)$ from (3.1) gives

$$
\begin{aligned}
\operatorname{Ric}^{B}(X, X) \phi^{B}-\operatorname{Hess}\left(\phi^{B}\right)(X, X)=\int_{F} & {\left[\widetilde{\operatorname{Ric}}_{\infty}^{M}(\bar{X}, \bar{X})+2\left(A_{\bar{X}}, A_{\bar{X}}\right)+(T \bar{X}, T \bar{X})\right.} \\
& \left.-\left(\frac{\bar{X} \phi^{M}}{\phi^{M}}-(\bar{X}, N)\right)^{2}\right] \phi^{M} d \operatorname{vol}_{F}
\end{aligned}
$$

Using (3.5),

$$
\begin{aligned}
\widetilde{\operatorname{Ric}}_{\infty}^{B}(X, X) \phi^{B}= & {\left[\operatorname{Ric}^{B}(X, X)-\frac{\operatorname{Hess}\left(\phi^{B}\right)(X, X)}{\phi^{B}}+\frac{\left(X \phi^{B}\right)^{2}}{\left(\phi^{B}\right)^{2}}\right] \phi^{B} } \\
= & \int_{F}\left[\widetilde{\operatorname{Ric}_{\infty}^{M}}(\bar{X}, \bar{X})+2\left(A_{\bar{X}}, A_{\bar{X}}\right)+(T \bar{X}, T \bar{X})\right. \\
& \left.-\left(\frac{\bar{X} \phi^{M}}{\phi^{M}}-(\bar{X}, N)\right)^{2}\right] \phi^{M} d \operatorname{vol}_{F} \\
& +\left(\int_{F}\left(\frac{\bar{X} \phi^{M}}{\phi^{M}}-(\bar{X}, N)\right) \phi^{M} d \operatorname{vol}_{F}\right)^{2}\left(\phi^{B}\right)^{-1} .
\end{aligned}
$$

We have

$$
\mathcal{L}_{\bar{X}}\left(\phi^{M} d \operatorname{vol}_{F}\right)=\left(\frac{\bar{X} \phi^{M}}{\phi^{M}}-(\bar{X}, N)\right) \phi^{M} d \operatorname{vol}_{F} .
$$

By assumption, $\frac{\bar{X} \phi^{M}}{\phi^{M}}-(\bar{X}, N)$ is constant on a fiber $F$. Then

$$
\begin{aligned}
\widetilde{\operatorname{Ric}}_{\infty}^{B}(X, X) \phi^{B} & =\int_{F}\left[\widetilde{\operatorname{Ric}}_{\infty}^{M}(\bar{X}, \bar{X})+2\left(A_{\bar{X}}, A_{\bar{X}}\right)+(T \bar{X}, T \bar{X})\right] \phi^{M} d \operatorname{vol}_{F} \\
& \geq \int_{F} \widetilde{\operatorname{Ric}}_{\infty}^{M}(\bar{X}, \bar{X}) \phi^{M} d \operatorname{vol}_{F} .
\end{aligned}
$$

If $\widetilde{\operatorname{Ric}}_{\infty}^{M}(\bar{X}, \bar{X}) \geq r g^{M}(\bar{X}, \bar{X})$ then (3.11) implies that $\widetilde{\operatorname{Ric}}_{\infty}^{B}(X, X) \geq r g^{B}(X, X)$. This proves Theorem 2.1 .

Now suppose that $\phi^{M}=1$. Equations (1.2) and (3.9) imply that

$$
\begin{aligned}
& {\widetilde{\operatorname{Ric}_{q}}}_{q}^{B}(X, X) \phi^{B} \\
& =\int_{F}\left[\operatorname{Ric}^{M}(\bar{X}, \bar{X})+2\left(A_{\bar{X}}, A_{\bar{X}}\right)+(T \bar{X}, T \bar{X})-\frac{1}{q}(\bar{X}, N)^{2}\right] d \operatorname{vol}_{F} \\
& \quad+\left(1-\frac{1}{q}\right)\left(-\int_{F}(\bar{X}, N)^{2} d \operatorname{vol}_{F}+\left(\int_{F}(\bar{X}, N) d \operatorname{vol}_{F}\right)^{2}\left(\phi^{B}\right)^{-1}\right) .
\end{aligned}
$$


As $(\bar{X}, N)=-\operatorname{Tr}(T \bar{X})$, we know that $(T \bar{X}, T \bar{X})-\frac{1}{q}(\bar{X}, N)^{2} \geq 0$. By assumption, $(\bar{X}, N)$ is constant on a fiber $F$. Then

$$
\begin{aligned}
& \quad \widetilde{\operatorname{Ric}}_{q}^{B}(X, X) \phi^{B} \\
& =\int_{F}\left[\operatorname{Ric}^{M}(\bar{X}, \bar{X})+2\left(A_{\bar{X}}, A_{\bar{X}}\right)+(T \bar{X}, T \bar{X})-\frac{1}{q}(\bar{X}, N)^{2}\right] d \operatorname{vol}_{F} \\
& \geq \int_{F} \operatorname{Ric}^{M}(\bar{X}, \bar{X}) d \operatorname{vol}_{F} . \\
& \text { If } \widetilde{\operatorname{Ric}}_{\infty}^{M}(\bar{X}, \bar{X}) \geq r g^{M}(\bar{X}, \bar{X}) \text { then } \\
& \\
& \quad \widetilde{\operatorname{Ric}}_{q}^{B}(X, X) \phi^{B} \geq r \int_{F} g^{M}(\bar{X}, \bar{X}) d \operatorname{vol}_{F}=r g^{B}(X, X) \phi^{B} .
\end{aligned}
$$

This proves Theorem 2.2 .

Example. Let $p: M \rightarrow B$ be a Riemannian submersion, with $M$ compact, whose fiber transport preserves the fiberwise metric up to multiplicative constants. Equivalently, the Riemannian metric $g$ on $M$ comes from starting with a submersion metric $g^{\prime}$ with totally geodesic fibers, along with a positive function $f \in C^{\infty}(B)$, and then multiplying the fiberwise metric of $g^{\prime}$ on $F_{b}$ by $f^{2}(b)$. One can think of $g$ as a generalized warped product metric.

Suppose that the fibers $F$ have nonnegative Ricci curvature. For $\epsilon>0$, let $g_{\epsilon}$ be the Riemannian metric on $M$ which comes from multiplying the fiberwise Riemannian metrics by $\epsilon^{2}$. Then as $\epsilon \rightarrow 0$, the metrics $g_{\epsilon}$ have Ricci curvatures that are uniformly bounded below. Explicitly, let $\bar{X}$ be the horizontal lift of a vector field $X$ on $B$ and let $\bar{U}$ be a vertical vector field. Then as $\epsilon \rightarrow 0$, with the notation of [7, Chapter 9],

$$
\begin{aligned}
& \operatorname{Ric}_{\epsilon}^{M}(\bar{X}, \bar{X}) \sim p^{*} \operatorname{Ric}^{B}(X, X)-(T \bar{X}, T \bar{X})+\left(\bar{X}, \nabla \frac{M}{X} N\right), \\
& \operatorname{Ric}_{\epsilon}^{M}(\bar{X}, \bar{U}) \sim 0 \\
& \operatorname{Ric}_{\epsilon}^{M}(\bar{U}, \bar{U}) \sim \operatorname{Ric}^{F}(\bar{U}, \bar{U})+\epsilon^{2}\left((\widetilde{\delta} T)(\bar{U}, \bar{U})-\left(N, T_{\bar{U}} \bar{U}\right)\right) .
\end{aligned}
$$

(The terms on the right-hand side of (3.15) are evaluated with respect to the metric $g_{1}$.) This is an example of a collapse with Ricci curvature bounded below, to which Theorem 2.2 applies.

For another example, let $M$ be a compact Riemannian manifold on which a Lie group $G$ acts isometrically and effectively. Suppose that the $G$-action on $M$ has a single orbit type and put $B=G \backslash M$. Then there is a natural Riemannian submersion $p: M \rightarrow B$. As the orbits of the $G$-action on $M$ are all $G$-diffeomorphic to a homogeneous space $G / H$, and $G / H$ has a unique $G$-invariant volume form up to constants, it follows that the fiber transport of the Riemannian submersion preserves measures up to constants. Hence Theorem 2.2 applies. 


\section{Proof of Theorem 3}

We refer to [15] for the definition of the measured Gromov-Hausdorff topology.

To prove Theorem 3.1, we just apply the warped product construction of the proof of Theorem 1.1 to $S^{q} \times B$.

Let $\left\{M_{i}, g_{i}\right\}_{i=1}^{\infty}$ be a sequence as in the statement of Theorem 3.2. We may assume that $\lim _{i \rightarrow \infty}\left(M_{i}, g_{i}, d \operatorname{vol}_{i}\right)=(X, \mu)$ in the measured Gromov-Hausdorff topology. If $q=0$ then $X$ is a smooth manifold with a $C^{1, \alpha}$-regular metric $g^{X}$ and after taking a subsequence and applying diffeomorphisms, we may assume that $\left(M_{i}, g_{i}\right)$ converges to $\left(X, g^{X}\right)$ in the $C^{1, \alpha}$-topology (see, for example, [18]). In this case, the theorem follows from Lemma 1.1.

Suppose that $q>0$. By saying that $X$ is a manifold, we mean that in the construction of $X$ as a quotient space $\widehat{X} / O(N)$ [16], the action of $O(N)$ on the manifold $\widehat{X}$ has a single orbit type. Then $X$ has the structure of a smooth manifold with a $C^{1, \alpha}$-regular pair $\left(g^{X}, \phi^{X}\right)$.

For any $\epsilon>0$, we can apply smoothing results of Abresch and others [11, Theorem 1.12] to obtain new metrics $g_{i}(\epsilon)$ with

$$
\begin{aligned}
e^{-\epsilon} g_{i} \leq g_{i}(\epsilon) & \leq e^{\epsilon} g_{i}, \\
\left|\nabla_{g_{i}}-\nabla_{g_{i}(\epsilon)}\right| & \leq \epsilon, \\
\left|\nabla_{g_{i}(\epsilon)}^{k} \operatorname{Riem}\left(M_{i}, g_{i}(\epsilon)\right)\right| & \leq C_{k}(N, \epsilon, \Lambda),
\end{aligned}
$$

where the constants are uniform. We can also assume that $\operatorname{Ric}\left(M_{i}, g_{i}(\epsilon)\right) \geq$ $(r-\epsilon) g_{i}(\epsilon)$ [13, Remark 2, p. 51]. (See [21, Theorem 2.1] for a similar statement about sectional curvature.) For small $\epsilon$, let $B(\epsilon)$ be a Gromov-Hausdorff limit of a subsequence of $\left\{\left(M_{i}, g_{i}(\epsilon)\right)\right\}_{i=1}^{\infty}$. We relabel the subsequence as $\left\{\left(M_{i}, g_{i}(\epsilon)\right)\right\}_{i=1}^{\infty}$. From [11, Proposition 4.9], for large $i$, there is a small $C^{2}$-perturbation $g_{i}^{\prime}(\epsilon)$ of $g_{i}(\epsilon)$ which is invariant with respect to a $N i l$-structure. In particular, we may assume that $\operatorname{Ric}\left(M_{i}, g_{i}^{\prime}(\epsilon)\right) \geq(r-2 \epsilon) g_{i}^{\prime}(\epsilon)$. Now $\left(M_{i}, g_{i}^{\prime}(\epsilon)\right)$ is the total space of a Riemannian submersion $M_{i} \rightarrow B(\epsilon)$ with infranil fibers and affine holonomy. Let $\left(g_{i}^{B(\epsilon)}, \phi_{i}^{B(\epsilon)}\right)$ denote the induced metric and measure on $B(\epsilon)$. As the fiber transport of the Riemannian submersion preserves the affine-parallel volume forms of the fibers, up to constants, Theorem 2.2 implies that $\widetilde{\operatorname{Ric}_{q}}\left(B(\epsilon), g_{i}^{B(\epsilon)}, \phi_{i}^{B(\epsilon)}\right) \geq$ $(r-2 \epsilon) g_{i}^{B(\epsilon)}$. Varying $i$ and $\epsilon$, we can extract a subsequence of $\left\{\left(B(\epsilon), g_{i}^{B(\epsilon)}, \phi_{i}^{B(\epsilon)}\right)\right\}$ with $i \rightarrow \infty$ and $\epsilon \rightarrow 0$ that converges in the $C^{1, \alpha}$-topology to $\left(X, g^{X}, \phi^{X}\right)$. The theorem now follows from Lemma 1.3.

\section{Proof of Theorem 4}

Let $s$ be a segment from $t_{0} \in T_{0}$ to $t \in T$, with length $l(s)>u_{3}$ and arclength parameter $u$. By definition, $s$ is length-minimizing. We can decompose the measure $\phi d \operatorname{vol}_{M}$ on $A\left(u_{1}, u_{4}\right)$ as $\phi$ area $_{s}(u) d u \mu(s)$, where $\mu$ is a measure on the 
space $\mathcal{S}$ of distinct segments $s$ that make up $A\left(u_{1}, u_{4}\right), d u$ is the length measure along a segment $s$ and $\operatorname{area}_{s}(u)$ is the relative size of the transverse Riemannian area density along $s$, as measured with respect to the fan of segments. Let $h$ denote the trace of the second fundamental form $\Pi$ of a level set of constant distance from $T_{0}$. (With our conventions, the boundary of the unit ball in $\mathbb{R}^{n}$ has positive mean curvature.) Differentiating along $s$, with respect to $u$, gives

$$
\partial_{u} \ln \left(\phi(u) \operatorname{area}_{s}(u)\right) \equiv \frac{\partial_{u}\left(\phi(u) \operatorname{area}_{s}(u)\right)}{\phi(u) \operatorname{area}(u))}=h(u)+\partial_{u} \ln \phi(u)
$$

and

$$
\partial_{u}^{2} \ln \left(\phi(u) \operatorname{area}_{s}(u)\right)=\partial_{u} h(u)+\partial_{u}^{2} \ln \phi(u) .
$$

From the Riccati equation for $\Pi$,

$$
\partial_{u} h(u)=-\operatorname{Tr}\left(\Pi^{2}\right)-\operatorname{Ric}\left(\partial_{u}, \partial_{u}\right) \leq-\operatorname{Ric}\left(\partial_{u}, \partial_{u}\right) .
$$

Then

$$
\partial_{u}^{2} \ln \left(\phi(u) \operatorname{area}_{s}(u)\right) \leq-\widetilde{\operatorname{Ric}}_{\infty}\left(\partial_{u}, \partial_{u}\right) \leq-r .
$$

Hence for any $c \in \mathbb{R}$,

$$
\partial_{u}^{2}\left(\ln \left(\phi(u) \operatorname{area}_{s}(u)\right)+\frac{r}{2} u^{2}-c u\right) \leq 0 .
$$

Fix $s$ and put

$$
\begin{gathered}
a(u)=\phi(u) \operatorname{area}_{s}(u), \\
\widehat{a}(u)=e^{-\frac{r}{2} u^{2}+c u}, \\
v\left(u_{1}, u_{2}\right)=\int_{u_{1}}^{u_{2}} a(u) d u
\end{gathered}
$$

and

$$
\widehat{v}\left(u_{1}, u_{2}\right)=\int_{u_{1}}^{u_{2}} \widehat{a}(u) d u
$$

Then (5.5) says that

$$
\frac{d^{2}}{d u^{2}} \ln \left(\frac{a}{\widehat{a}}\right) \leq 0
$$

i.e. that $\ln \left(\frac{a}{\hat{a}}\right)$ is concave in $u$.

Lemma 2. If $\frac{v\left(u_{2}, u_{3}\right)}{\widehat{v}\left(u_{2}, u_{3}\right)} \leq \frac{v\left(u_{1}, u_{2}\right)}{\widehat{v}\left(u_{1}, u_{2}\right)}$ then $\frac{a\left(u_{3}\right)}{\widehat{a}\left(u_{3}\right)} \leq \frac{v\left(u_{2}, u_{3}\right)}{\widehat{v}\left(u_{2}, u_{3}\right)}$.

Proof. Suppose that

$$
\frac{a\left(u_{3}\right)}{\widehat{a}\left(u_{3}\right)}>\frac{v\left(u_{2}, u_{3}\right)}{\widehat{v}\left(u_{2}, u_{3}\right)}=\frac{\int_{u_{2}}^{u_{3}} \frac{a(u)}{\widehat{a}(u)} \widehat{a}(u) d u}{\int_{u_{2}}^{u_{3}} \widehat{a}(u) d u} .
$$


If $\frac{a\left(u_{2}\right)}{\widehat{a}\left(u_{2}\right)} \geq \frac{a\left(u_{3}\right)}{\widehat{a}\left(u_{3}\right)}$ then the concavity of $\ln \left(\frac{a}{\widehat{a}}\right)$ implies that

$$
\frac{a\left(u_{3}\right)}{\widehat{a}\left(u_{3}\right)} \leq \frac{\int_{u_{2}}^{u_{3}} \frac{a(u)}{\widehat{a}(u)} \widehat{a}(u) d u}{\int_{u_{2}}^{u_{3}} \widehat{a}(u) d u},
$$

which is a contradiction. Thus

$$
\frac{a\left(u_{2}\right)}{\widehat{a}\left(u_{2}\right)}<\frac{a\left(u_{3}\right)}{\widehat{a}\left(u_{3}\right)} .
$$

With the concavity of $\ln \left(\frac{a}{\widehat{a}}\right),(5.13)$ implies that $\frac{a(u)}{\widehat{a}(u)}$ is decreasing in $u$ for $u<u_{2}$ and so

$$
\frac{\int_{u_{1}}^{u_{2}} \frac{a(u)}{a} \widehat{a}(u)}{\int_{u_{1}}^{u_{2}} \widehat{a}(u) d u}<\frac{a\left(u_{2}\right)}{\widehat{a}\left(u_{2}\right)} .
$$

The concavity of $\ln \left(\frac{a}{\hat{a}}\right)$ and (5.13) also imply that

$$
\frac{a\left(u_{2}\right)}{\widehat{a}\left(u_{2}\right)}<\frac{\int_{u_{2}}^{u_{3}} \frac{a(u)}{\widehat{a}(u)} \widehat{a}(u) d u}{\int_{u_{2}}^{u_{3}} \widehat{a}(u) d u} .
$$

Thus we have

$$
\frac{\int_{u_{1}}^{u_{2}} \frac{a(u)}{\widehat{a}(u)} \widehat{a}(u) d u}{\int_{u_{1}}^{u_{2}} \widehat{a}(u) d u}<\frac{a\left(u_{2}\right)}{\widehat{a}\left(u_{2}\right)}<\frac{\int_{u_{2}}^{u_{3}} \frac{a(u)}{\bar{a}(u)} \widehat{a}(u) d u}{\int_{u_{2}}^{u_{3}} \widehat{a}(u) d u},
$$

which contradicts the assumption.

Lemma 3. If $\frac{v\left(u_{2}, u_{3}\right)}{\widehat{v}\left(u_{2}, u_{3}\right)} \leq \frac{v\left(u_{1}, u_{2}\right)}{\widehat{v}\left(u_{1}, u_{2}\right)}$ then for $u_{4} \in\left(u_{3}, l(s)\right), \frac{v\left(u_{3}, u_{4}\right)}{\widehat{v}\left(u_{3}, u_{4}\right)} \leq \frac{v\left(u_{2}, u_{3}\right)}{\widehat{v}\left(u_{2}, u_{3}\right)}$.

Proof. For $u \in\left(u_{3}, l(s)\right)$, put

$$
F(u)=\ln \left(\frac{v\left(u_{3}, u\right)}{\widehat{v}\left(u_{3}, u\right)} / \frac{v\left(u_{2}, u_{3}\right)}{\widehat{v}\left(u_{2}, u_{3}\right)}\right) .
$$

Then

$$
F^{\prime}(u)=\frac{a(u)}{v\left(u_{3}, u\right)}-\frac{\widehat{a}(u)}{\widehat{v}\left(u_{3}, u\right)}=\frac{\widehat{a}(u)}{v\left(u_{3}, u\right)}\left[\frac{a(u)}{\widehat{a}(u)}-\frac{v\left(u_{3}, u\right)}{\widehat{v}\left(u_{3}, u\right)}\right] .
$$

Lemma 2 implies that if $F(u) \leq 0$ then $F^{\prime}(u) \leq 0$. We can extend $F(u)$ smoothly to $u=u_{3}$, with

$$
F\left(u_{3}\right)=\ln \left(\frac{a\left(u_{3}\right)}{\widehat{a}\left(u_{3}\right)} / \frac{v\left(u_{2}, u_{3}\right)}{\widehat{v}\left(u_{2}, u_{3}\right)}\right) .
$$

By Lemma 2, $F\left(u_{3}\right) \leq 0$. It follows that $F(u) \leq 0$ for all $u \in\left(u_{3}, l(s)\right)$, which proves the lemma.

We have

$$
\frac{\operatorname{vol}_{\phi}\left(A\left(u_{2}, u_{3}\right)\right)}{\operatorname{vol}_{\phi}\left(A\left(u_{1}, u_{2}\right)\right)}=\frac{\int_{\mathcal{S}} \frac{v_{s}\left(u_{2}, u_{3}\right)}{v_{s}\left(u_{1}, u_{2}\right)} v_{s}\left(u_{1}, u_{2}\right) d \mu(s)}{\int_{\mathcal{S}} v_{s}\left(u_{1}, u_{2}\right) d \mu(s)} .
$$


Put

and

$$
\mathcal{S}^{\prime}=\left\{s \in S: \frac{v_{s}\left(u_{2}, u_{3}\right)}{v_{s}\left(u_{1}, u_{2}\right)}<\frac{\widehat{v}\left(u_{2}, u_{3}\right)}{\widehat{v}\left(u_{1}, u_{2}\right)}\right\}
$$

$$
T^{\prime}=\bigcup_{s \in S^{\prime}} s
$$

We claim that (1.8) is satisfied. If it is not satisfied, put $\mathcal{S}^{\prime \prime}=\mathcal{S}-\mathcal{S}^{\prime}$ and $T^{\prime \prime}=T-T^{\prime}$. Then

$$
\frac{\operatorname{vol}_{\phi}\left(T^{\prime \prime} \cap A\left(u_{1}, u_{2}\right)\right)}{\operatorname{vol}_{\phi}\left(A\left(u_{1}, u_{2}\right)\right)}>\frac{\operatorname{vol}_{\phi}\left(A\left(u_{2}, u_{3}\right)\right)}{\operatorname{vol}_{\phi}\left(A\left(u_{1}, u_{2}\right)\right)}\left(\frac{\widehat{v}\left(u_{2}, u_{3}\right)}{\widehat{v}\left(u_{1}, u_{2}\right)}\right)^{-1} .
$$

However, from the definition of $T^{\prime \prime}$,

$$
\begin{aligned}
\operatorname{vol}_{\phi}\left(A\left(u_{2}, u_{3}\right)\right) & \geq \operatorname{vol}_{\phi}\left(T^{\prime \prime} \cap A\left(u_{2}, u_{3}\right)\right)=\int_{\mathcal{S}^{\prime \prime}} \frac{v_{s}\left(u_{2}, u_{3}\right)}{v_{s}\left(u_{1}, u_{2}\right)} v_{s}\left(u_{1}, u_{2}\right) d \mu(s) \quad(5.24) \\
& \geq \int_{\mathcal{S}^{\prime \prime}} \frac{\widehat{v}\left(u_{2}, u_{3}\right)}{\widehat{v}\left(u_{1}, u_{2}\right)} v_{s}\left(u_{1}, u_{2}\right) d \mu(s)=\frac{\widehat{v}\left(u_{2}, u_{3}\right)}{\widehat{v}\left(u_{1}, u_{2}\right)} \operatorname{vol}_{\phi}\left(T^{\prime \prime} \cap A\left(u_{1}, u_{2}\right)\right),
\end{aligned}
$$

which contradicts $(5.23)$.

If there is a cutpoint along $s$, with respect to its basepoint in $T_{0}$, at $u_{c} \in$ $\left(u_{3}, u_{4}\right)$ then we put $v_{s}\left(u_{3}, u_{4}\right)=\int_{u_{3}}^{u_{c}} a_{s}(u) d u$, and otherwise we put $v_{s}\left(u_{3}, u_{4}\right)=$ $\int_{u_{3}}^{u_{4}} a_{s}(u) d u$. Using Lemma 3 ,

$$
\frac{\operatorname{vol}_{\phi}\left(T^{\prime} \cap A\left(u_{3}, u_{4}\right)\right)}{\operatorname{vol}_{\phi}\left(T^{\prime} \cap A\left(u_{2}, u_{3}\right)\right)}=\frac{\int_{\mathcal{S}^{\prime}} \frac{v_{s}\left(u_{3}, u_{4}\right)}{v_{s}\left(u_{2}, u_{3}\right)} v_{s}\left(u_{2}, u_{3}\right) d \mu(s)}{\int_{\mathcal{S}^{\prime}} v_{s}\left(u_{2}, u_{3}\right) d \mu(s)} \leq \frac{\widehat{v}_{s}\left(u_{3}, u_{4}\right)}{\widehat{v}_{s}\left(u_{2}, u_{3}\right)} .
$$

This proves the first part of the theorem.

Suppose that there is a number $r \in \mathbb{R}$ so that for each tube $T$ and $c \in \mathbb{R}$ satisfying (1.7), there is a subtube $T^{\prime}$ satisfying the properties of the theorem. Given $m \in M$ and a unit vector $v \in T_{m} M$, let $T_{0}$ be a hypersurface passing through $m$ such that $T_{m}\left(T_{0}\right)=v^{\perp}$ and the second fundamental form of $T_{0}$ at $m$ vanishes. Let $s$ be a minimizing segment with $s(0)=m$ and $s^{\prime}(0)=v$. From (5.1),

$$
\left.\frac{d}{d u}\right|_{u=0}(\ln (\phi(u) \operatorname{area}(u))=v(\ln \phi) .
$$

From (5.2) and the Riccati equation,

$$
\left.\frac{d^{2}}{d u^{2}}\right|_{u=0}\left(\ln (\phi(u) \operatorname{area}(u))=-\widetilde{\operatorname{Ric}}_{\infty}(v, v)\right.
$$

Put $c_{0}=v(\ln \phi)$ and $r_{0}=\widetilde{\operatorname{Ric}}_{\infty}(v, v)$. Then for small $u$,

$$
\ln (\phi(u) \operatorname{area}(u)) \sim \text { const. }+c_{0} u-\frac{r_{0}}{2} u^{2} .
$$

For small $u_{1}<u_{2}<u_{3}<u_{4}$, we have

$$
\frac{v\left(u_{2}, u_{3}\right)}{v\left(u_{1}, u_{2}\right)} \sim \frac{\int_{u_{2}}^{u_{3}} e^{-\frac{r_{0}}{2} u^{2}+c_{0} u} d u}{\int_{u_{1}}^{u_{2}} e^{-\frac{r_{0}}{2} u^{2}+c_{0} u} d u}
$$


and

$$
\frac{v\left(u_{3}, u_{4}\right)}{v\left(u_{2}, u_{3}\right)} \sim \frac{\int_{u_{3}}^{u_{4}} e^{-\frac{r_{0}}{2} u^{2}+c_{0} u} d u}{\int_{u_{2}}^{u_{3}} e^{-\frac{r_{0}}{2} u^{2}+c_{0} u} d u}
$$

Take $T$ to be a small tube around $s$ (with small base $T_{0}$ ), take $u_{3}$ small relative to $u_{4}$ and take $c=c_{0}+\epsilon$ with $\epsilon>0$ small so that (1.7) holds. If there is to be a subtube $T^{\prime}$ such that (1.9) holds, for all such choices, then we must have $r_{0} \geq r$. This proves the theorem.

\section{Remarks}

1. If $M^{n}$ is compact and $\widetilde{\operatorname{Ric}}_{q} \geq r g$, with $q$ an integer greater than one, then Theorem 3.1 says that $(M, g, \phi)$ is the limit of a sequence of $(n+q)$-dimensional manifolds with Ricci curvature bounded below by $r$. As in the proof of Theorem 1.2, we can then apply standard results about manifolds with Ricci curvature bounded below, in order to obtain conclusions about $(M, g, \phi)$. For example, applying the Bishop-Gromov inequality to the $(n+q)$-dimensional manifolds and taking the limit, we obtain a Bishop-Gromov-type inequality for the measures of the distance balls in $M$. Namely, let $\operatorname{vol}_{\phi}$ denote the weighted measure. Then for $0<u_{1}<u_{2}, \frac{\operatorname{vol}_{\phi}\left(B_{u_{2}}\right)}{\operatorname{vol}_{\phi}\left(B_{u_{1}}\right)}$ is less than or equal to the corresponding quantity in the $(n+q)$-dimensional space form of Ricci curvature $r$. If $r>0$ then applying Myers' theorem to the $(n+q)$-dimensional manifolds and taking the limit, we obtain that $\operatorname{diam}(M) \leq \pi \sqrt{\frac{n+q-1}{r}}$. This gives alternative proofs of some results of Qian [20, Corollary 2 and Theorem 5] in the special case when $q$ is an integer greater than one. (The results of [20] are valid for all positive q.) One can also show that if $\widetilde{\operatorname{Ric}}_{q} \geq r g$ with $q \in(0, \infty)$ then $(M, g, \phi)$ satisfies the directional Bishop-Gromov inequality of $[8,($ A.2.2)] with respect to a model space of formal dimension $n+q$.

2. Similarly, if $q$ is an integer greater than one then there are Sobolev inequalities for the $(n+q)$-dimensional collapsing manifolds [6, Theorem 3, p. 397]. Applying these inequalities to functions that pullback from $M$, we obtain weighted Sobolev inequalities for $M$. Namely, put $V=\int_{M} \phi d \operatorname{vol}_{M}$. Given $\alpha, \beta \in[1, \infty)$ such that $\alpha \leq \frac{(n+q) \beta}{n+q-\beta}$, let $\Sigma(n+q ; \alpha, \beta)$ be the Sobolev constant of the standard $(n+q)$-sphere $S^{n+q}$, defined by

$$
\Sigma(n+q ; \alpha, \beta)=\sup \left\{\frac{\|f\|_{\alpha}}{\|d f\|_{\beta}}: f \in W^{1, \beta}\left(S^{n+q}\right), f \neq 0, \int_{S^{n+q}} f=0\right\}
$$


Then if $\widetilde{\operatorname{Ric}_{q}}(M, g, \phi) \geq \frac{n+q-1}{R^{2}} g$, we have

$$
\begin{gathered}
\left(\int_{M} f^{\alpha} \phi d \operatorname{vol}_{M}\right)^{\frac{1}{\alpha}} \leq \Sigma(n+q ; \alpha, \beta) R\left(\frac{V}{\operatorname{vol}\left(S^{n+q}\right)}\right)^{\frac{1}{\alpha}-\frac{1}{\beta}}\left(\int_{M}|\nabla f|^{\beta} \phi d \operatorname{vol}_{M}\right)^{\frac{1}{\beta}} \\
+V^{\frac{1}{\alpha}-\frac{1}{\beta}}\left(\int_{M} f^{\beta} \phi d \operatorname{vol}_{M}\right)^{\frac{1}{\beta}}
\end{gathered}
$$

for $f \in W^{1, \beta}(M)$. In the case $\beta=2$, these inequalities appeared in [3].

3. From the Bishop-Gromov-type inequalities, one can easily show that for any $q, D \in \mathbb{R}^{+}$and $r \in \mathbb{R}$, the space of Riemannian manifolds $(M, g)$ with a smooth positive probability measure $\phi d \operatorname{vol}_{M}$ satisfying $\widetilde{\operatorname{Ric}}_{q}(M, g, \phi) \geq r g$ and $\operatorname{diam}(M, g) \leq D$, taken modulo diffeomorphisms, is precompact in the measured Gromov-Hausdorff topology.

Since the relative volume in $\mathbb{R}^{n+q}$ of $B_{u_{2}}$ and $B_{u_{1}}$ is $\left(\frac{u_{2}}{u_{1}}\right)^{n+q}$, we cannot expect any Bishop-Gromov-type comparison theorem for the masses of balls in spaces with $\widetilde{\operatorname{Ric}}_{\infty}$ bounded below, i.e. when $q \rightarrow \infty$ in $\widetilde{\operatorname{Ric}}_{q}$. However, it is interesting that spaces with $\widetilde{\operatorname{Ric}}_{\infty} \geq r g$ for $r>0$ do admit isoperimetric inequalities [5].

4. It is an interesting question whether there is a good synthetic notion of a metric-measure space with Ricci curvature bounded below, in analogy to the notion of an Alexandrov space with curvature bounded below. See [8, Appendix $2]$ for discussion. It is clear from Theorem 3.1 that triples $(M, g, \phi)$ with $\widetilde{\operatorname{Ric}}_{q} \geq r g$ are examples of metric-measure spaces with generalized Ricci curvature bounded below by $r$, at least if $q$ is an integer greater than one.

There are various ways that one could try to extend the notion of Ricci curvature bounded below, from smooth metric-measure spaces to more general metricmeasure spaces. One could fix $q \in(0, \infty)$ and try to extend the notion of having $\widetilde{\operatorname{Ric}}_{q} \geq r g$. Or one could consider all $q$ simultaneously, and say in particular that a triple $(M, g, \phi)$ has generalized Ricci curvature bounded below by $r$ if $\widetilde{\operatorname{Ric}}_{q} \geq r g$ for some $q \in(0, \infty)$. Or one could consider a triple $(M, g, \phi)$ to have generalized Ricci curvature bounded below by $r$ if $\widetilde{\operatorname{Ric}}_{\infty} \geq r g$.

We note that there is a difference between having $\widetilde{\operatorname{Ric}_{q}} \geq r g$ for some $q \in$ $(0, \infty)$ and having $\widetilde{\operatorname{Ric}_{\infty}} \geq r g$. For example, if $r>0$ and $\widetilde{\operatorname{Ric}}_{q} \geq r g$ for some $q \in(0, \infty)$ then $M$ is compact [20, Theorem 5], whereas if $\widetilde{\operatorname{Ric}_{\infty}} \geq r g$ then $M$ can be noncompact (as in the case of $\mathbb{R}$ with $\phi(x)=e^{-\frac{r}{2} x^{2}}$.) It is also easy to see that triples $(M, g, \phi)$ with $\widetilde{\operatorname{Ric}}_{\infty} \geq 0$ generally do not satisfy the splitting principle.

If one does consider a triple $(M, g, \phi)$ with $\widetilde{R i c}_{\infty} \geq r g$ to be an admissible space with generalized Ricci curvature bounded below by $r$ then one has a large class of examples. For instance, from this viewpoint it would be reasonable to say that flat $\mathbb{R}^{n}$ with the measure $e^{-V} d x_{1} \ldots d x_{n}$ has nonnegative generalized Ricci curvature if $V$ is any convex function on $\mathbb{R}^{n}$. 


\section{References}

[1] M. Anderson and J. Cheeger, $C^{\alpha}$-Compactness for Manifolds with Ricci Curvature and Injectivity Radius Bounded Below, J. Diff. Geom. 35 (1992), 265-281.

[2] C. Ané, S. Blachère, D. Chafaï, P. Fougères, I. Gentil, F. Malrieu, C. Roberto and G. Scheffer, Sur les Inégalités de Sobolev Logarithmiques, Panoramas et Synthèses 10, Soc. Math. de France, Paris, 2000.

[3] D. Bakry, L'Hypercontractivité et son Utilisation en Théorie des Semigroupes, in: Lectures on Probability Theory (Saint-Flour, 1992), 1-114, Lecture Notes in Math. 1581, Springer, Berlin, 1994.

[4] D. Bakry and M. Émery, Diffusions Hypercontractives, in: Séminaire de probabilités XIX, 1983/84, 177-206, Lecture Notes in Math. 1123, Springer, Berlin, 1985.

[5] D. Bakry and M. Ledoux, Lévy-Gromov's Isoperimetric Inequality for an InfiniteDimensional Diffusion Generator, Inv. Math. 123 (1996), 259-281.

[6] P. Bérard, From Vanishing Theorems to Estimating Theorems: the Bochner Technique Revisited, Bull. Amer. Math. Soc. 19 (1988), 371-406.

[7] A. Besse, Einstein Manifolds, Ergebnisse der Mathematik und ihrer Grenzgebiete (3), vol. 10, Springer-Verlag, Berlin, 1987.

[8] J. Cheeger and T. Colding, On the Structure of Spaces with Ricci Curvature Bounded Below I, J. Diff. Geom. 46 (1997), 406-480.

[9] J. Cheeger and T. Colding, On the Structure of Spaces with Ricci Curvature Bounded Below II, J. Diff. Geom. 54 (2000), 13-35.

[10] J. Cheeger and T. Colding, On the Structure of Spaces with Ricci Curvature Bounded Below III, J. Diff. Geom. 54 (2000), 37-74.

[11] J. Cheeger, K. Fukaya and M. Gromov, Nilpotent Structures and Invariant Metrics on Collapsed Manifolds, J. Amer. Math. Soc. 5 (1992), 327-372.

[12] J. Cheeger and D. Gromoll, The Splitting Theorem for Manifolds of Nonnegative Ricci Curvature, J. Diff. Geom. 6 (1971), 119-128.

[13] X. Dai, G. Wei and R. Ye, Smoothing Riemannian Metrics with Ricci Curvature Bounds, Manuscripta Math. 90 (1996), 49-61.

[14] J.-P. Demailly, Estimations $L^{2}$ pour l'Opérateur $\bar{\partial}$ d'un Fibré Vectoriel Holomorphe SemiPositif Au-Dessus d'une Variété Kählérienne Complète, Ann. Sci. École Norm. Sup. (4) 15 (1982), 457-511.

[15] K. Fukaya, Collapsing of Riemannian Manifolds and Eigenvalues of Laplace Operator, Inv. Math. 87 (1987), 517-547.

[16] K. Fukaya, A Boundary of the Set of the Riemannian Manifolds with Bounded Curvatures and Diameters, J. Diff. Geom. 28 (1988), 1-21.

[17] M. Gromov, Metric Structures for Riemannian and Non-Riemannian Spaces, Birkhäuser, Boston, 1999

[18] A. Kasue, A Convergence Theorem for Riemannian Manifolds and Some Applications, Nagoya Math. J. 114 (1989), 21-51.

[19] M. Ledoux, The Geometry of Markov Diffusion Generators, Ann. Fac. Sci. Toulouse Math. (6) 9 (2000), 305-366.

[20] Z. Qian, Estimates for Weighted Volumes and Applications, Quart. J. Math. Oxford Ser. (2) 48 (1997), 235-242.

[21] X. Rong, On the Fundamental Groups of Manifolds of Positive Sectional Curvature, Ann. of Math. 143 (1996), 397-411.

[22] E. Witten, Supersymmetry and Morse Theory, J. Diff. Geom. 17 (1982), 661-692. 
John Lott

Department of Mathematics

University of Michigan

Ann Arbor, MI 48109-1109

USA

e-mail: lott@umich.edu

(Received: November 19, 2002)

To access this journal online:

(20) http://www.birkhauser.ch 